\title{
Implementation of Approximations of Belief Functions for Fusion of ESM Reports within the DSm Framework
}

\author{
Pascal Djiknavorian ${ }^{1}$, Pierre Valin ${ }^{2}$, Dominic Grenier ${ }^{1}$ \\ ${ }^{1}$ Electrical and Computer Engineering Dept. \\ Université Laval, Québec, Canada \\ Email: dominic.grenier@gel.ulaval.ca \\ ${ }^{2} \mathrm{C} 2$ Decision Support Systems section \\ Defence R\&D Canada - Valcartier, Québec, Canada \\ Email: Pierre.Valin@drdc-rddc.gc.ca
}

\begin{abstract}
Electronic Support Measures consist of passive receivers which can identify emitters which, in turn, can be related to platforms that belong to 3 classes: Friend, Neutral, or Hostile. Decision makers prefer results presented in STANAG 1241 allegiance form, which adds 2 new classes: Assumed Friend, and Suspect. DezertSmarandache (DSm) theory is particularly suited to this problem, since it allows for intersections between the original 3 classes. However, as we know, the DSm hybrid combination rule is highly complex to execute and requires high amounts of resources. We have applied and studied a Matlab implementation of Tessem's $k$-l-x, Lowrance's Summarization and Simard's approximation techniques in the DSm theory for the fusion of ESM reports. Results are presented showing that we can improve on the time of execution while maintaining or getting better rates of good decisions in some cases.
\end{abstract}

Keywords: Dezert-Smarandache Theory, ESM, approximations, Belief functions.

\section{Introduction}

In terms of classification, the Dezert-Smarandache theory (DSmT) can become quite useful, especially for the direct resolution of classification for cases of hierarchical classes structures. For instance, we have the case of the allegiance classification structure suggested by STANAG 1241 where a structure of five classes ( 3 main classes and 2 derived classes) is required. The DSmT is able to output to any of those classes without modifications to its fusion process.

However, this example is still a simple one and both DSmT theories, with or without approximation, can solve it quite easily, which wouldn't be the case for classification problems of higher dimension. By dimension we mean the cardinal of the frame of discernment. In fact, the DSmT can become highly complex and computationally prohibitive as soon as we reach a dimension of 6 . That is a classification of a problem having six main classes and up to, in the worst case scenario, a total of 7,828,353 possible derived classes.

Various avenues of research have been tried to avoid or address this complexity problem [10, 13, 18]. However, even just counting the number of possible classes is still an active problem in mathematics known as the Dedekind problem, or the problem of counting antichains $[9,18]$.

In this paper, we study the use of an approximation technique to restrain the staggering amount of data that the DSmT can generate in its fusion process. More specifically we have chosen Tessem's klx approximation technique [4], Lowrance's Summarization [19], Simard's and al technique $[3,7,8]$ and used them into the DSmT with the DSm hybrid combination rule (DSmH). We have also experimented with the fusion process while using the approximation technique and compared it to the case without an approximation technique to analyze how it affects the quality of the decision process. More specifically, we will compare the good decision rate in the two cases, with and without the use of approximation.

\subsection{Realistic Case Study}

Electronic Support Measures (ESM) consist of passive receivers which can identify emitters coming from a small bearing angle, which, in turn, can be related to platforms that belong to 3 classes: either Friend (F), Neutral (N), or Hostile $(\mathrm{H})$. Decision makers prefer results presented in STANAG 1241 allegiance form, which adds 2 classes: Assumed Friend (AF), and Suspect (S).

The DSm theory is particularly suited to this problem, since it allows for intersections between the original 3 classes of allegiance. In this way an intersection of Friend and Neutral can lead to an Assumed Friend, and an intersection of Hostile and Neutral can lead to a Suspect. This structure of allegiances will be referred to as STANAG allegiance [11].

Figure 1 displays a visual representation of a possible interpretation of STANAG allegiance in DSmT. We can see that even though the input consists only of three classes, we are able to give an output into five classes. For example, here we have the class 'Suspect', which could be the result obtained after fusing 'Hostile' with 'Neutral'. We also have the class 'Assumed Friend', which could be the result obtained after fusing 'Friend' with 'Neutral'. Note that this case example has the intersection $\mathrm{F} \cap \mathrm{H}=\varnothing$, the null set, which is a constraint in DSm, leading to the use of its hybrid rule. This case example would be relevant for peacekeeping missions where Hostile and Friendly forces aren't 
likely to be close one to another. We will be working on that case, with $\mathrm{F} \cap \mathrm{H}=\varnothing$.

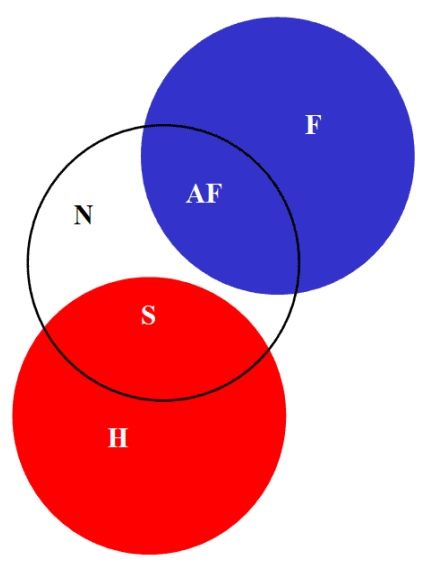

Figure 1. Venn diagram for the STANAG allegiances.

\section{Dezert-Smarandache Theory}

The DSm theory uses the language of masses assigned to each declaration from a sensor (in our case, the ESM sensor). In DSm theory, all unions and intersections are allowed for a declaration. For our case of cardinality $3, \Theta=$ $\left\{\theta_{1}, \theta_{2}, \theta_{3}\right\}$, with $|\Theta|=3, D^{\Theta}$ is still of manageable size, namely has a cardinality of 19 [10]. In DSm theory, a constraint like the one that was imposed by Figure 1, namely that $\mathrm{F} \cap \mathrm{H} \equiv \theta_{1} \cap \theta_{3}=\varnothing$ is treated by the DSm hybrid combination rule (DSmH) below:

$$
m(A)=\phi(A)\left[S_{1}(A)+S_{2}(A)+S_{3}(A)\right]
$$

The reader is referred to a series of books $[10,13,17]$ on DSm theory for lengthy descriptions of the meaning of this formula (note that the function $\phi$ is not to be confused with the empty set). A three-step approach was proposed in [12], which is used here. The incoming sensor reports are either: Friend $\left(\mathrm{F}=\theta_{1}\right)$, Neutral $\left(\mathrm{N}=\theta_{3}\right)$ or Hostile $\left(\mathrm{H}=\theta_{3}\right)$, Figure 1 has the interpretation of the five classes:

$$
\begin{aligned}
& \text { Friend }=\left\{\theta_{1}-\theta_{1} \cap \theta_{2}\right\} \\
& \text { Hostile }=\left\{\theta_{3}-\theta_{3} \cap \theta_{2}\right\} \\
& \text { Assumed Friend }=\left\{\theta_{1} \cap \theta_{2}\right\} \\
& \text { Suspect }=\left\{\theta_{2} \cap \theta_{3}\right\} \\
& \text { Neutral }=\left\{\theta_{2}-\theta_{1} \cap \theta_{2}-\theta_{3} \cap \theta_{2}\right\}
\end{aligned}
$$

As in [15], we call STANAG-probability the pignistic probability assigned to the five classes shown by equations (2) to (6). We use the general pignistic transform, as shown by [10] or equation (7), to obtain the probability values of the sets used in those equations.

$$
P\{A\}=\sum_{X \in D^{\ominus}} \frac{C_{\mathcal{M}}(X \cap A) m(X)}{C_{\mathcal{M}}(X)}
$$

Where $C_{M}(\mathrm{~A})$, is the DSm cardinal of a set A. It accounts for the total number of partitions. Each of these partitions possesses a numeric weight equal to one. That weight, identical for each part makes them all equal. The DSm cardinal is used in the generalized pignistic transformation equation to redistribute the masse of a set $\mathrm{A}$ among all its partitions B such that B is included or equal to A.

\section{Approximation technique}

\subsection{K-l-x approximation}

The k-l-x approximation technique developed by Tessem [4] is designed to approximate Basic Probability Assignment (BPA) or mass function in Dempster-Shafer Theory (DST). Since DSm theory works directly with BPAs, applying the $\mathrm{k}-1-\mathrm{x}$ approximation technique to the DSmH is quite straightforward and can be done without any changes.

This algorithm for approximation of BPAs involves three parameters: $\mathrm{k}$ the minimum number of focal elements to be kept, 1 the maximum number of focal elements to be kept and $\mathrm{x}$ the maximum threshold on the sum of the lost masses. It can be summarized as follows:

\section{Select the $\mathrm{k}$ focal elements with highest masses;}

2. While the sum of their masses is less than 1-x, and while their number is less than 1 , add the next focal element with highest mass.

\subsection{Simard's and al. approximation}

This truncation scheme [3, 7, 8] has had many minor variations over time. Similarly to k-l-x approximation, it was conceived to approximate BPA or mass function in DST. And as in k-l-x, we were able to transfered it to the DSm framework. Variants exist but all focus on preferentially keeping fused propositions with the smallest lengths (lowest cardinality) after passing 2 thresholding steps. The rule therefore involves 3 parameters: BPAmax, BPAmin and Nmax. It retains fused propositions according to the following rules:

1. All fused propositions with $\mathrm{BPA}>\mathrm{BPA}_{\max }$ are kept (thresholding step 1)

2. All fused propositions with $\mathrm{BPA}<\mathrm{BPA}_{\min }$ are discarded (thresholding step 2) 
3. If the number of retained propositions in step 1 is smaller than $\mathrm{N}_{\max }$, retain by decreasing BPA, propositions of length 1 , then if the number of retained propositions is smaller than $\mathrm{N}_{\text {max }}$, retain by decreasing BPA, propositions of length 2 , and so on for 3 ...

4. If the number of retained propositions is still smaller than $\mathrm{N}_{\max }$, retain propositions by decreasing BPA regardless of length.

\subsection{Lowrance's approximation}

Similar to the k-1-x procedure, the summarization method [19] (inspired by the summarization operation described in Bauer's research [5]) leaves the best-valued focal elements of the mass function under consideration unchanged. The numerical values of the remaining focal elements are accumulated and assigned to the set-theoretic union of the corresponding subsets of $\Theta$. Here again, the technique was conceived to approximate BPA or mass function in DST, and we were able to transfer it to the DSm framework.

\subsection{Implementation of approximations}

The information coming from the sensor is a simple belief function giving a mass to an allegiance and the remaining mass to ignorance. The combination itself combines two belief functions, one is the information from the sensor at time $t$, the other contains past information within combination result from time $t-1$. The fusion process is realized dynamically. Since the information to combine from the sensor is a simple belief function the approximation is applied on the result of the combination.

\section{A typical simulation scenario}

The pre-requisites that a typical scenario must address are: (1) to be able to adequately represent the known ground truth, (2) to contain sufficient countermeasures (or missassociations) to be realistic and to test the robustness of the theories, (3) to only provide partial knowledge about the ESM sensor declaration, which therefore contains uncertainty, (4) to be able to show stability under countermeasures, yet (5) to be able to switch allegiance when the ground truth does so.

The following scenario parameters have therefore been chosen accordingly: (1) ground truth is FRIEND for the first 50 iterations of the scenario and HOSTILE for the last 50 , (2) the number of correct associations is $80 \%$, corresponding to countermeasures appearing $20 \%$ of the time, in a randomly selected sequence, (3) the ESM declaration has a mass (confidence value in Bayesian terms) of 0.8 , with the rest of the mass being assigned to the ignorance (the full set of elements, namely $\Theta$ ).

This scenario will be the one addressed in the next section, while a Monte-Carlo study is described in the subsequent sections. Each Monte-Carlo run corresponds to a different realization using the above scenario parameters, but with a different random seed. The chosen scenario is depicted in Figure 2.

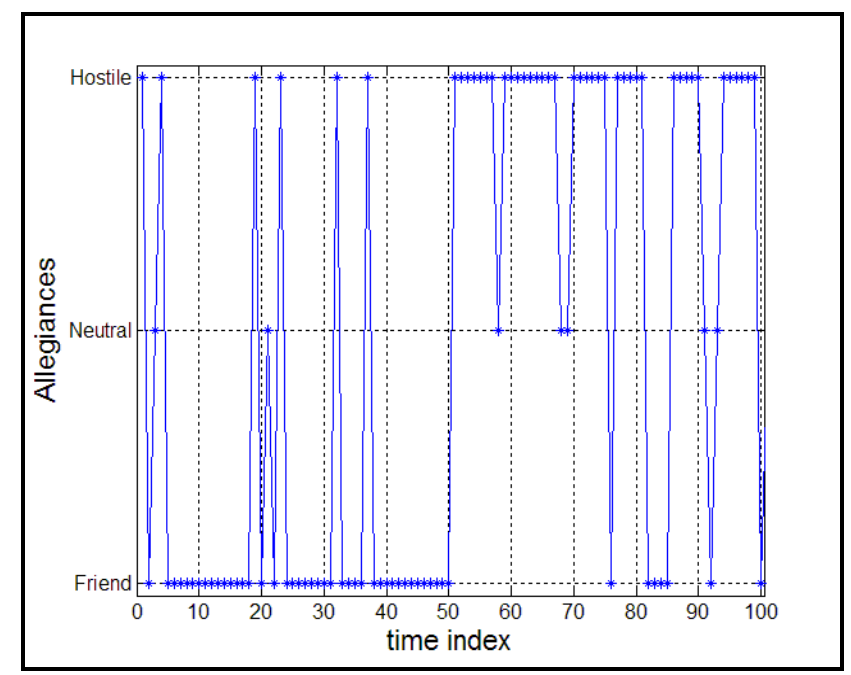

Figure 2. Chosen scenario.

Roughly $80 \%$ of the time the ESM declares the correct allegiance according to ground truth, and the remaining $20 \%$ is roughly equally split between the other two allegiances. Note that these percentages of occurrences are from a statistical point of view only, so that in the long run a large amount of randomly generated scenarios would amount to these ratios. There is an allegiance switch at the 50th time index, and the selected randomly selected seed in the above generated scenario generates a rather unusual sequence of 4 false Friend declarations starting at time index 82 (when actually Hostile is the ground truth).

\subsection{Results for the simulated scenario}

Before presenting the results, it should be noted that the original form of the DSmH tends to accumulates masses to intersections as is the case for any rule based on conjunction [14]. An ad hoc solution exists [3, 7, 8], and consists in renormalizing after each fusion step by giving a value to the complete ignorance which can never be below a certain factor (chosen here to be 0.04 as research in [14] shows that this value is appropriate for this case while being high enough to avoid the accumulation but still low enough not to interfere with the combination's performances). That solution was originally developed to the well-known problem of DST combination, which tends to be overly optimistic, which in turn prevents it to react quickly to changes of allegiances. For more on the behavior of the DSmH on similar cases the reader is referred to $[14,15$, 16], as we are focused on exploring the effect of approximations on DSm here.

Since the whole idea behind using DSm was to present the results to the decision maker in the STANAG allegiance format, the result of Figure 3 would be used. For the DSmH 
[10], it was suggested to use the Generalized Pignistic Probability, which is based on the pignistic transformation $[6,10]$, in order to make a decision on a singleton belonging to the input ESM-allegiance.

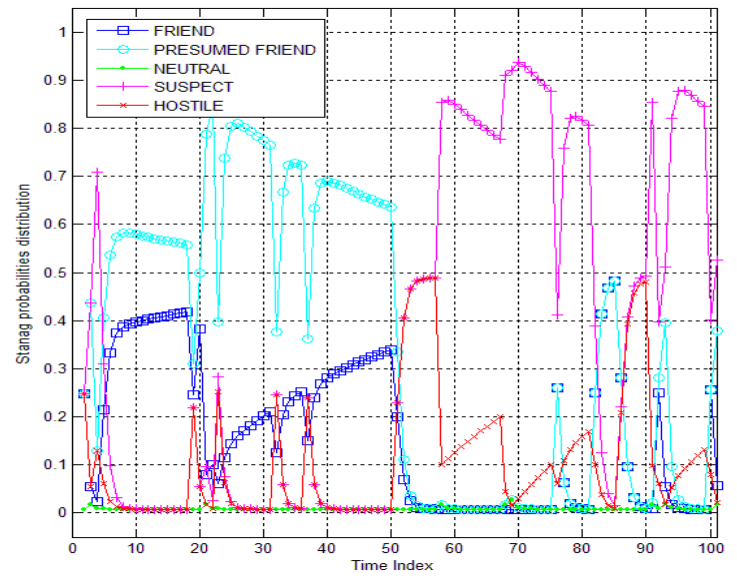

Figure 3. DSmH result for the chosen scenario.

The decision maker would clearly be informed that missassociations have occurred, since Assumed Friend dominates for the first 50 time indices and Suspect for the latter 50. The Friend declarations starting at time index 82 cause confusion, as it should. The change in allegiance at time index 50 is detected quickly. What is even more important is that $\mathrm{F}$ and $\mathrm{AF}$ are clearly preferred for the first 50 time indexes and $\mathrm{S}$ and $\mathrm{H}$ for the last 50 , as they should.

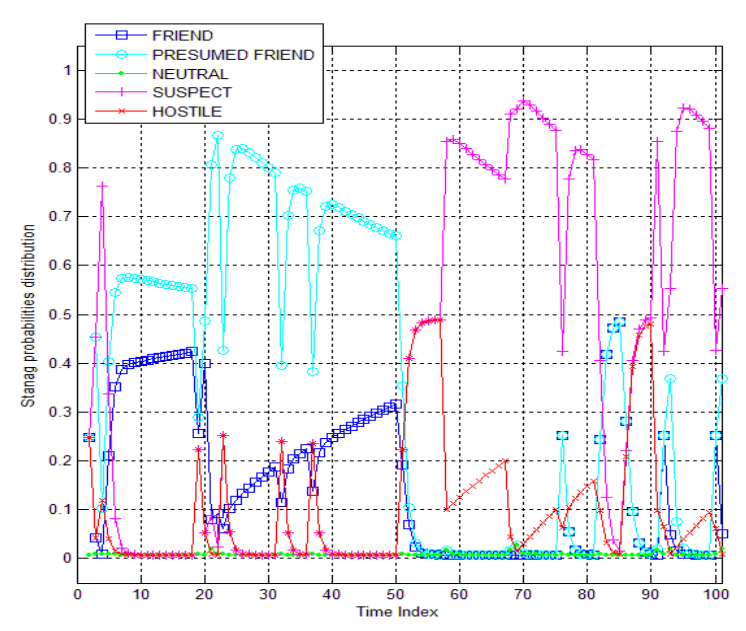

Figure 4. Approximated DSmH result for the same scenario with $\mathrm{k}-\mathrm{l}-\mathrm{x}=(5,6,0.2)$

We can gather from Figure 4 and Figure 5 that the DSmH and the approximated DSmH have very similar behaviors. In fact, one has to look at the figures very closely to perceive the differences. We can see that in the first half of the approximated version, the assumed friend allegiance is slightly favored to the friend allegiance. Near the end of the scenario the hostile allegiance is favored to the suspect allegiance. However, in both cases, even if the smallness of the change could possibly affect our decision, the STANAG-probability still seems to stay within the same type of allegiance in the sense that a friend and a target of assumed friend allegiance would both inspire a friendly response on our part. The same can be said for a target of suspect or hostile allegiance that would both inspire a hostile or defensive response on our part. In short, we can easily proceed with the approximation and still be able to make the same decision the same way.

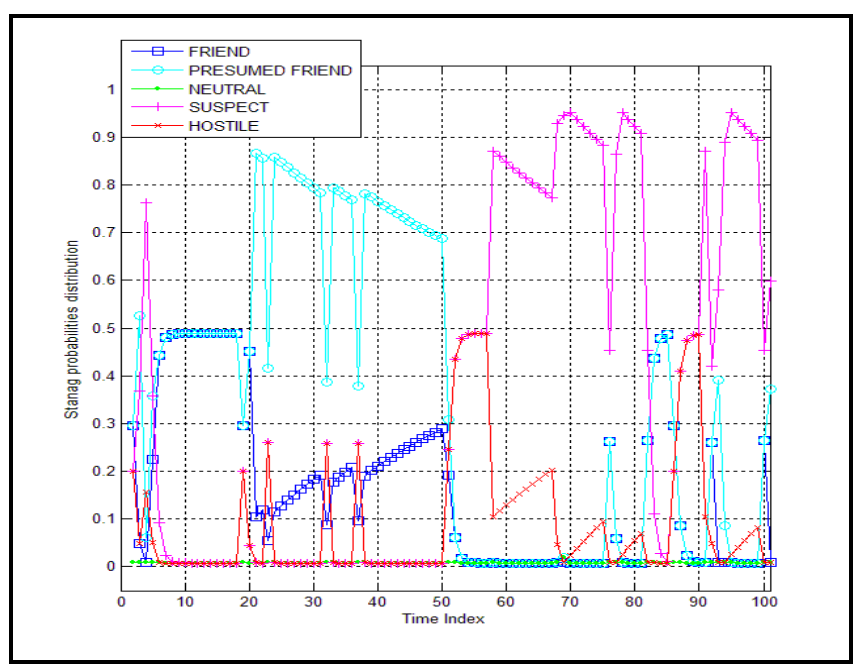

Figure 5. Approximated DSmH result for the same scenario with $\mathrm{k}-\mathrm{l}-\mathrm{x}=(3,6,0.2)$

\subsection{Effects of varying the $k-1-x$ parameters}

We've realized the scenario for various values of $\mathrm{k}-\mathrm{l}-\mathrm{x}$ for $\mathrm{k}$ $\in[3,10], 1 \in[6,12]$ and $x \in[0.2,0.4]$. For the cases where we had $\mathrm{k}=8$, no changes in 1 and $\mathrm{x}$ had impact, and compared to the DSmH, we've only noticed a very small variation at the start and end of the simulated scenario. For the cases where we had $\mathrm{k}=6$, no changes in 1 and $\mathrm{x}$ had impact and compared to $\mathrm{DSmH}$, there was only very little variation in value throughout the scenario. The same is true for the cases with $\mathrm{k}=5$, with the Figure 4 showing the results for that case. The amplitude of the variation between DSmH and the approximated version continues to increase as the $\mathrm{k}$ value diminishes.

We finally begin to notice small changes with $\mathrm{x}=0.2$ as opposed to 0.3 or 0.4 when we reach $\mathrm{k}=4$. However, the impact of having $\mathrm{x}$ at 0.2 is small and contained at the start of the scenario, where it gives more weight to the suspect class at the expense of the hostile class. For the cases with $\mathrm{k}=3$, the impact of the change on $\mathrm{x}$ going to 0.2 was more significant and lasted throughout most of the scenario's duration. Also, while for cases of $\mathrm{k} \in[4,8]$ the behavior of the curves were all very similar one to another, when we reach $\mathrm{k}=3$, we observe a partial loss of smoothness, hence a more reactive behavior toward countermeasures and allegiance change. Figure 5 shows the case of the simulated 
scenario for an approximated DSmH with $\mathrm{klx}=(3,6,0.2)$. Note that in all our experimentations for our chosen scenario the 1 parameter never had any visible impact.

\section{Monte-Carlo Simulations with k-l-x approximation}

Although a special case such as the one described in the previous section offers valuable insight, one might question if the conclusions from that one scenario pass the test of multiple Monte-Carlo scenarios. This question is answered in this section.

In order to expend the parameter space, we have realized the simulations of the current section to 80 and $90 \%$ for the ESM certainty, and with an ESM confidence at $80 \%$ and an ignorance threshold at 0.04 as before. The number of Monte-Carlo runs was set to 100 . The randomly generated ESM stream of reports used for both the DSmH and the approximated DSmH are all the same so that we can freely compare the effects of the use of the approximation, and the impact of the variation of its parameters.

As for the choice of a the graphical display to highlight the results of our simulations, we went with the rate of good decisions, where a good decision is as we have mentioned earlier, when we conclude to be friendly toward a friendly behaving target, when the ground truth is of class friend. A friendly-behaving target is a target that is concluded to be a friend or an assumed friend. We also have a good decision when we conclude to be hostile toward a hostile behaving target, when the ground truth is of class hostile. A hostilebehaving target is a target that is concluded to be a hostile or a suspect. A decision is made by taking the set of maximum STANAG-probability.

\subsection{Effects of varying the $\mathrm{k}-\mathrm{l}-\mathrm{x}$ parameters}

Simulations were done on a computer with a Phenom II 955 processor with $8 \mathrm{~GB}$ of memory. We should keep in mind that it is the relative time of execution which is important here. For figures 7 to 11 , the simulations had a value of $80 \%$ for the ESM certainty and the value of the $\mathrm{x}$ parameter was maintained at 0.2 since changing it had no impact on good decision rate.

Figure 7 and Figure 8 show us the effect of the approximation from the good decision rate point of view when compared with the DSmH case from Figure 6. Like for the typical simulated scenario from previous section, 1 had no visible impact, and $\mathrm{x}$ had a limited impact only as the $\mathrm{k}$ parameter went below 4 . As for the $\mathrm{k}$ parameter, it started having an impact when we reached 6 , where the impact was on only three iterations. As the $\mathrm{k}$ parameter reaches 5 , a very slight positive impact throughout the whole simulation can be seen. As for $\mathrm{k}=4$ and $\mathrm{k}=3$, we have a slight deterioration of the good decision rate but it is still very small and rather insignificant considering the gain in time execution as Figure 10 shows us. For the cases with an ESM confidence at 90\%, all the approximated results, have no significant impact on the good decision rate, except with $\mathrm{klx}=(3,8,0.2)$ where we had minimal impact.

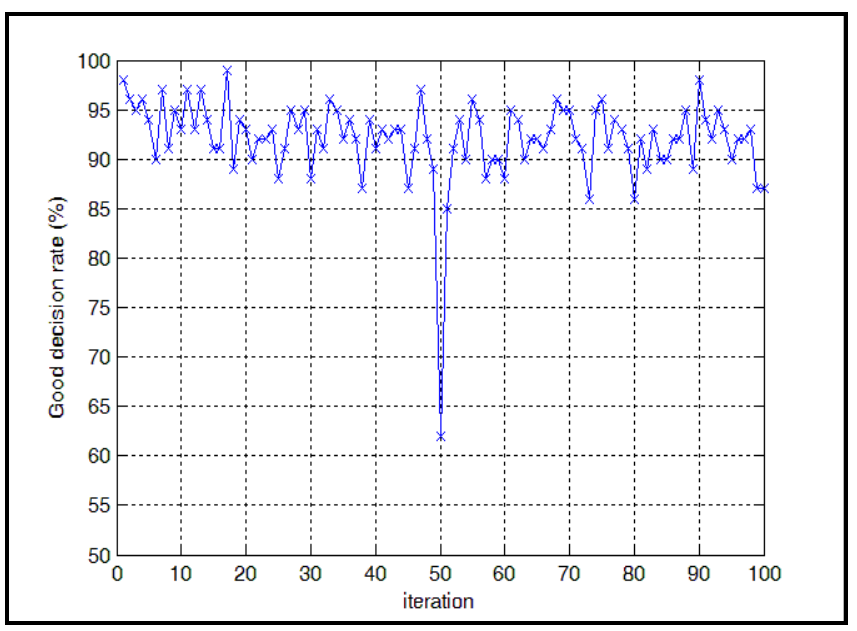

Figure 6. DSmH result after 100 Monte-Carlo runs.

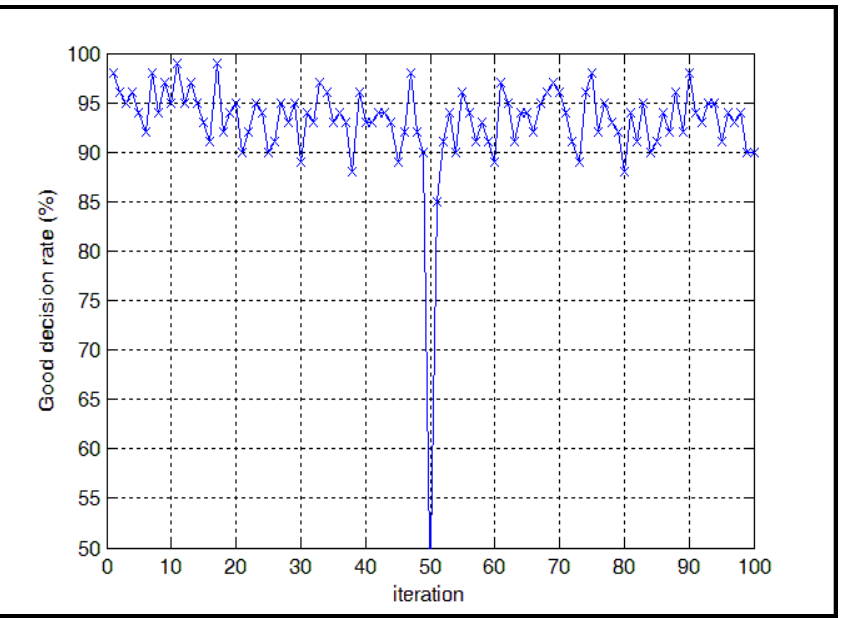

Figure 7. Approximated DSmH result with $\mathrm{k}-\mathrm{l}-\mathrm{x}=(5,8$, 0.2 ) for the same Monte-Carlo simulation.

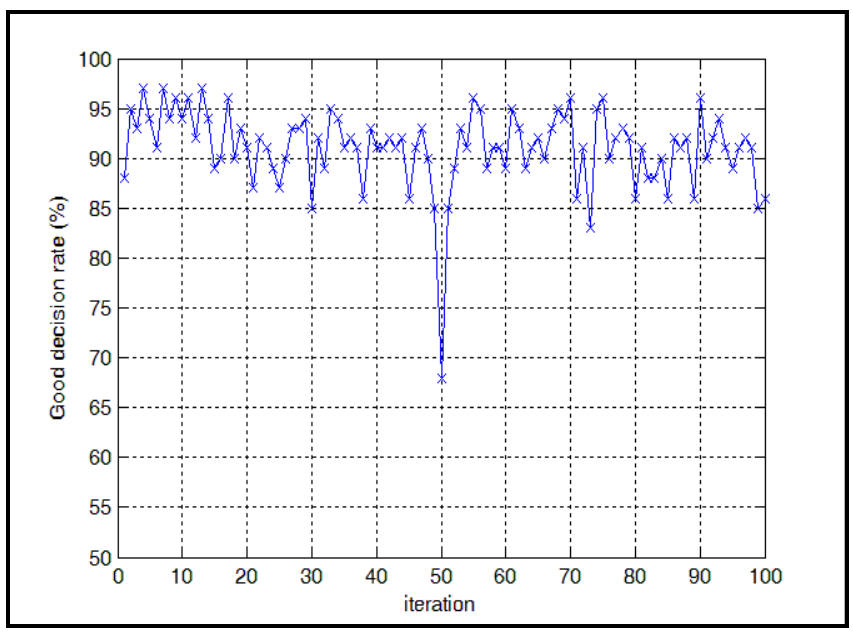

Figure 8. Approximated DSmH result with $\mathrm{k}-1-\mathrm{x}=(3,8,0.2)$ for the same Monte-Carlo simulation. 
We have the time of execution versus $\mathrm{k}$ and 1 parameters from the klx approximation technique on Figure 9 and Figure 10. Specifically, Figure 9 has the curve of the time of execution of the combination and approximation process only. The x-y plane, valued at 325.97 seconds on Figure 9 indicates the time from which the approximation process provides a higher gain in time than the time it consumes. It is the time of execution of the $\mathrm{DSmH}$ without approximation.

We can see that the $\mathrm{k}$ parameter has to reach 5 before we start seeing an improvement. Before that value, the approximation takes more time to execute than it helps us gain. We can achieve a $30 \%$ improvement on time of execution when we reach $\mathrm{k}=3$. The parameter 1 has no impact on time. The absence of impact of the 1 parameter is suspected to be caused by the fact that this simulated scenario case uses simple support functions as inputs.

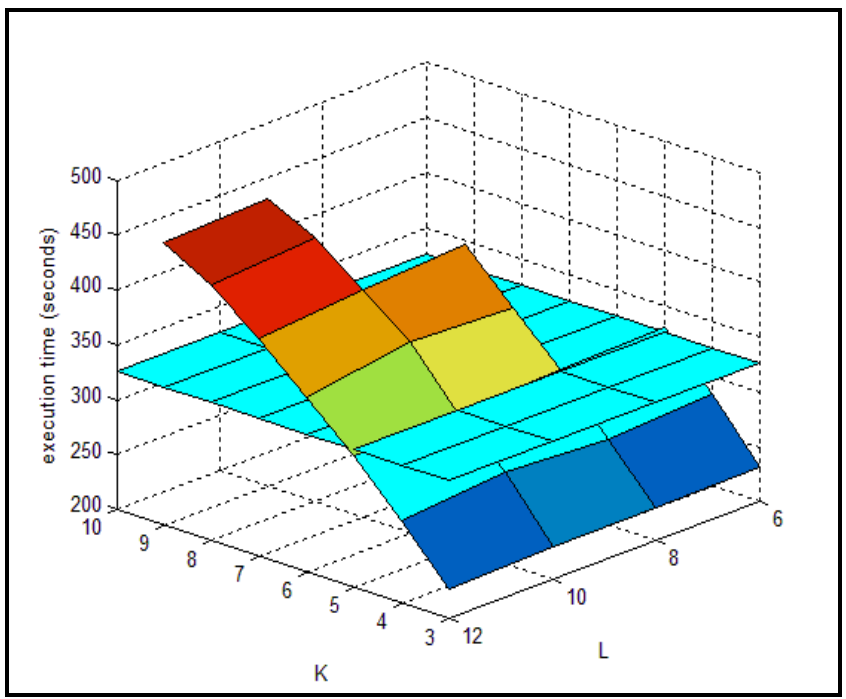

Figure 9. Execution time for the combination and approximation processes.

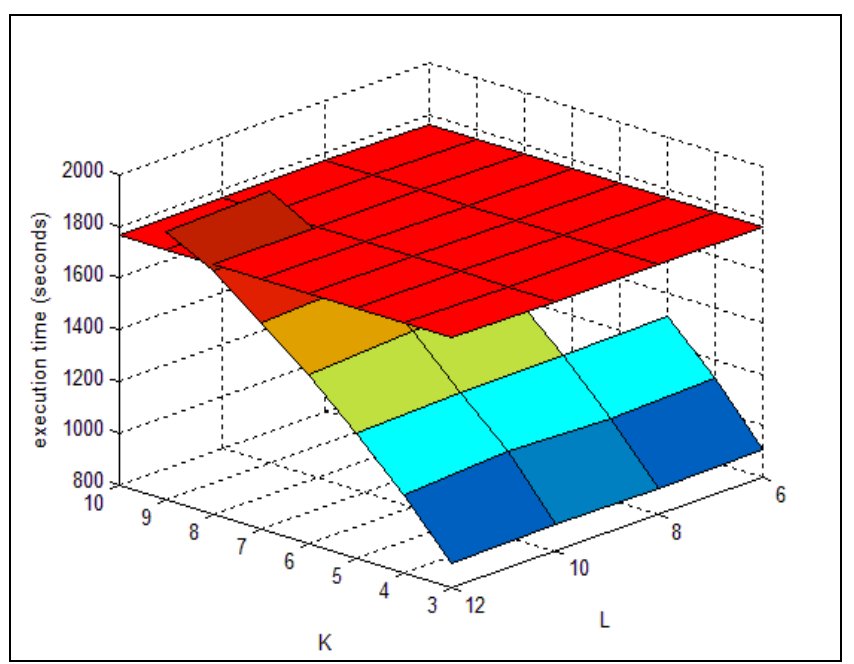

Figure 10. Execution time for the whole simulation.
In Figure 10, we have the curve of the time of execution for the whole simulation which, on top of the combination and approximation processes, includes the generalized pignistic transformation (GPT) which is used in the decision process. Above $95 \%$ of the extra time of execution, when compared to figure 10 , is composed of the GPT.

In Figure 10, the $x-y$ plane, representing the time of execution of the simulation without approximation, is valued at 1767.6 seconds. We can see that we can have a $50 \%$ reduction in time of execution when we reach $\mathrm{k}=3$ and that 1 has no impact. As we compare Figure 9 and Figure 10 , we see that the GPT is the step that benefits the most from the approximation process.

\section{Monte-Carlo simulations using various approximation rules}

In order to expend the analysis furthermore, we have realized the simulations of the current section with MonteCarlo runs set to 1000. Also, we've expended the analysis to Simard's summarization, and Simard's truncation techniques with the same stream of reports to fuse. Hence, both the DSmH and the approximated DSmH will have the same dataset so that we can freely compare the effects of the use of the approximation, and the impact of the variation of its parameters.

Figure 11, which shows results using Lowrance's approximation technique lets us see the inability of the technique to get better good decision rates than the non approximated combination. The following figures shows that k-l-x, and Simard's Truncation are both able to get, depending on the chosen parameters, better results of good decision rates, than the scenario without approximation.

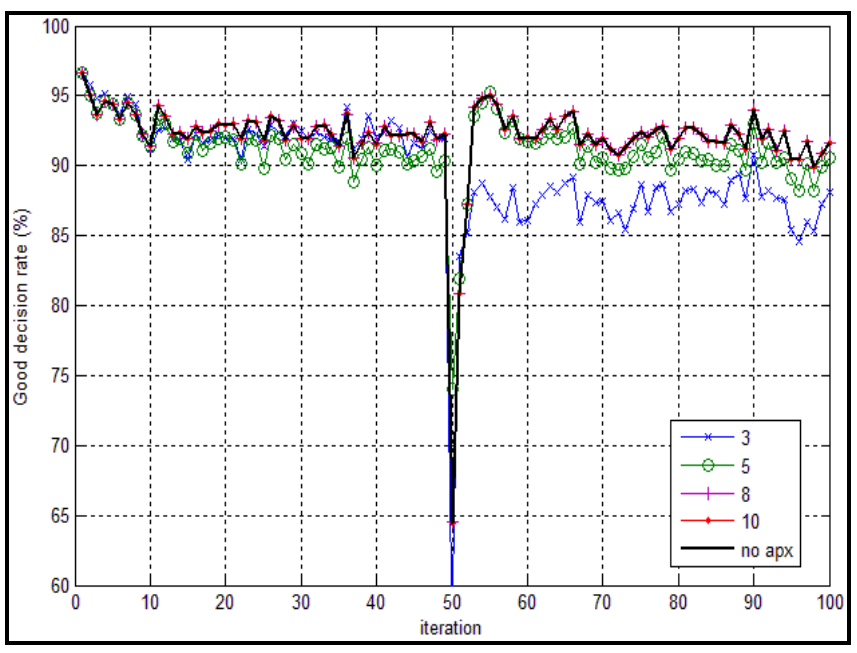

Figure 11. DSmH using Lowrance's apx. (3/5/8/10). 


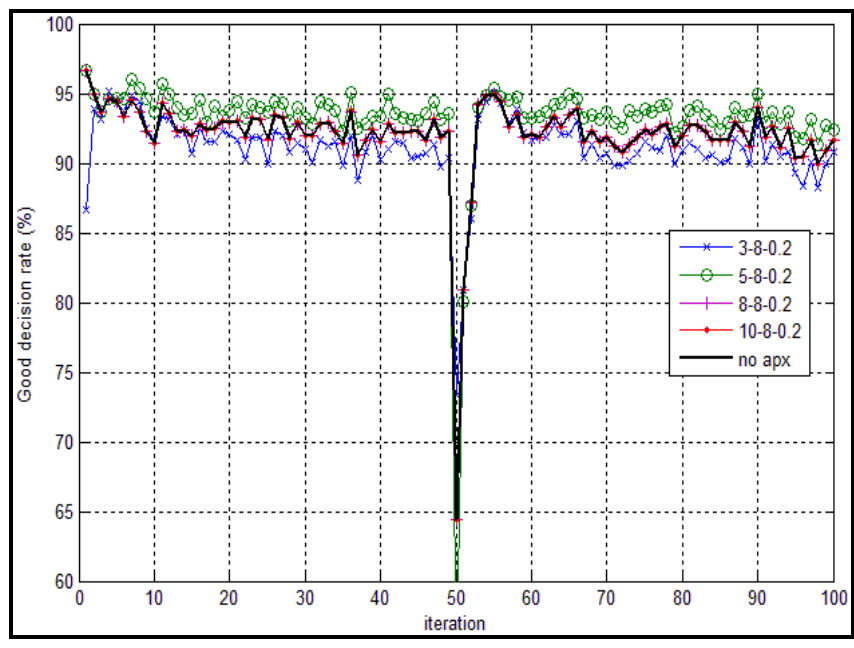

Figure 12. DSmH using k-1-x apx. (3/5/8/10-8-0.2).

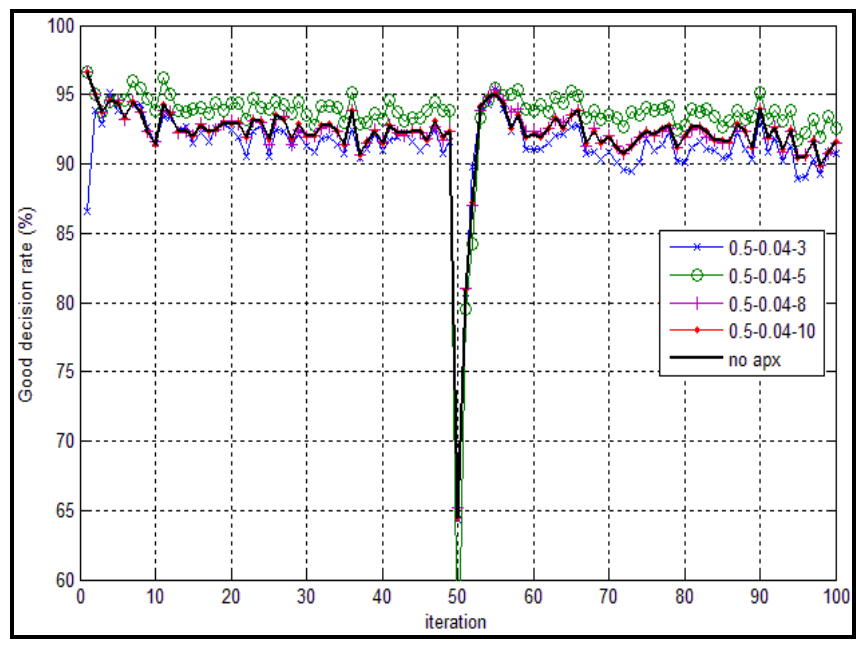

Figure 13. DSmH using Simard's apx. (0.5-0.04-3/5/8/10).

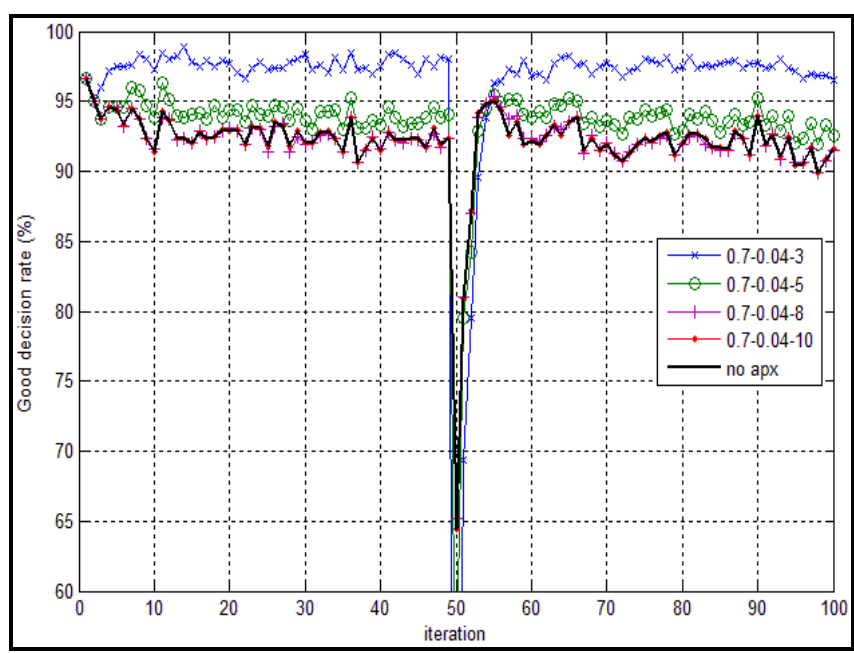

Figure 14. DSmH using Simard's apx. (0.7-0.04-3/5/8/10).

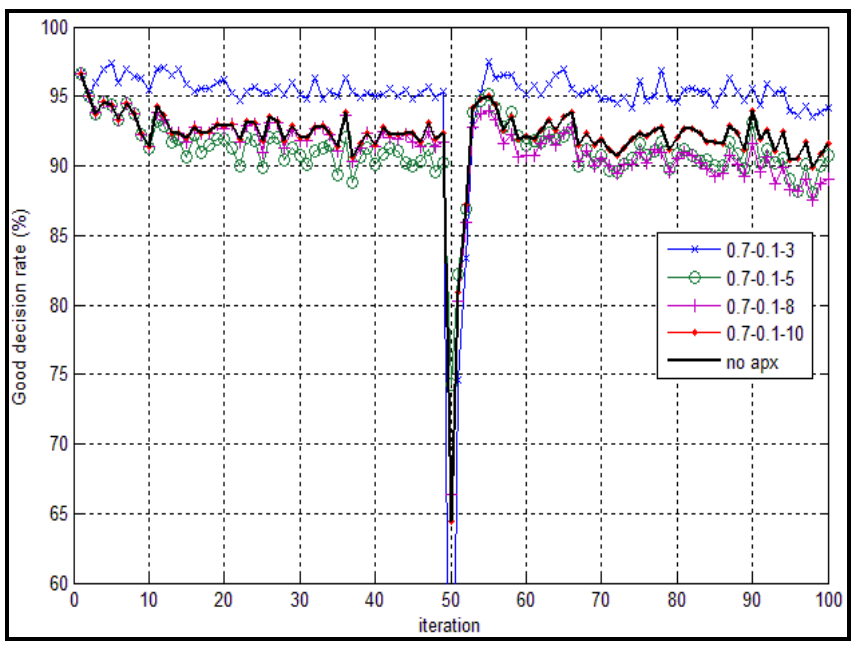

Figure 15. DSmH using Simard's apx. (0.7-0.1-3/5/8/10).

About the mean time of execution of the combination and approximation step for realistic scenario, we have found that for a parameter ' $\mathrm{K}$ ' below, or equal, to 5, we were able to execute faster than without approximation. And when looking at previous figures, we see that, too low $(\mathrm{K} \sim 3)$, the approximation isn't as good as without approximation, and that at a value of 5, we were always at higher good decision rates than the case without approximation.

So not only we have found a case executing faster than without approximation, but we've also found ourselves a case where it performs better in terms of good decision rate. That is for approximation techniques different from Lowrance's, and limited, until proven differently, to this case, and for $\mathrm{DSmH}$.

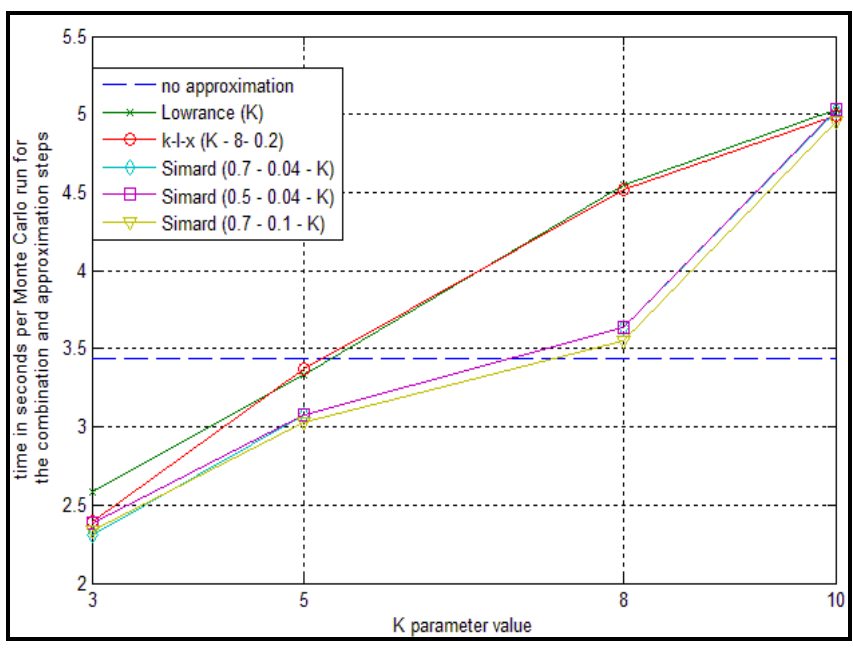

Figure 16. Combination and approximation execution times in seconds per Monte-Carlo run. 


\section{Conclusions}

The previous sections display the behavior for different cases of klx approximation on the same simulated ESM data (see Figure 6, Figure 7, and Figure 8). It also shows the time of execution of each of those simulations. From those results we can conclude that we can successfully attain the same good decision rate with DSmH as with an approximated DSmH for the chosen scenario, while achieving lower times of execution including the time to approximate when we reach a certain level of approximation. Those results are confirmed by the experimentation done on another simulated dataset lasting for 1000 Monte-Carlo runs. (see Figure 12)

We've also explored the behavior of Lowrance's summarization approximation and Simard's truncation methods while using the same dataset also on a thousand Monte-Carlo runs. From what we can see on Figure 11, the summarization is able, with the careful choice of its parameter, to reach good decision rate of the combination rule without approximation, however, it seems to be rarely able to do better and can do much worst. Simard's truncation method (see Figure 13, Figure 14 and Figure 15) on the other hand is able to get around 5\% better good decision rates, depending on the choice of the approximation parameters. It can also get the same rates or a little less than the combination rule without approximation.

When considering results of time of execution as shown on Figure 16 we gather that, while being able to execute faster than the combination rule without approximation, we can get better decision rates. The ' $\mathrm{K}$ ' parameter value of approximation of each rule, when at 3 or 5 , gave us highest decision rates for Simard's truncation method or k-l-x approximation technique. Note that some times, parameter $\mathrm{K}$ had to be set at 3 , other times at 5 , depending on chosen technique and the other parameters, to reach highest decision rate.

Future work considered includes the exploration of the use of Bauer's D1 approximation [5] in DSmT. Even if it adds to the number of operations and in the complexity of the system, it would be interesting to see if the gain acquired by approximating is sufficient to counter the increase in complexity. We are also interested to see if it is able to give even better good decision rates than the other methods of approximation.

\section{References}

[1] A.P. Dempster,. Upper and lower probabilities induced by a multivalued mapping, Ann. Math. Statist. 38, 1967. pp. 325-339.
[2] G. Shafer, A Mathematical Theory of Evidence, Princeton Univ. Press, Princeton, NJ, 1976.

[3] M.A. Simard, P. Valin and E. Shahbazian Fusion of ESM, Radar, IFF and other Attribute Information for Target Identity Estimation and a Potential Application to the Canadian Patrol Frigate, AGARD 66th Symposium on Challenge of Future EW System Design, 18-21 October 1993, Ankara (Turkey), AGARD-CP-546, pp. 14.1-14.18.

[4] B. Tessem, Approximations for efficient computation in the theory of evidence, Artificial Intelligence, vol. 61, pp. 315-329, June 1993.

[5] M. Bauer, Approximation Algorithms and Decision Making in the Dempster-Shafer Theory of Evidence-An Empirical study, International Journal of Approximate Reasoning, vol. 17, no. 2-3, pp. 217-237, 1997.

[6] Ph. Smets, Data Fusion in the Transferable Belief Model, Proceedings of the 3rd International Conference on Information Fusion, Fusion 2000, Paris, July 10-13, 2000, pp PS21-PS33.

[7] D. Boily, and P. Valin, Truncated Dempster-Shafer Optimization and Benchmarking, in Sensor Fusion: Architectures, Algorithms, and Applications IV, SPIE Aerosense 2000, Orlando, Florida, April 2428 2000, Vol. 4051, pp. 237-246.

[8] D. Boily, and P. Valin, Optimization and Benchmarking of Truncated Dempster-Shafer for Airborne Surveillance, NATO Advanced Study Institute on Multisensor and Sensor Data Fusion, Pitlochry, Scotland, United Kingdom, June 25 - July 7 2000. Kluwer Academic Publishers, NATO Science Series, II. Mathematics Physics and Chemistry - Vol. 70, pp. 617-624.

[9] R. Fidytek, A.W. Mostowski, R. Somla and A. Szepietowski. Algorithms counting monotone Boolean functions, Information Processing Letters, Vol. 79, Issue 5, pp. 203-209, 15 September 2001.

[10] F. Smarandache, J. Dezert, editors. Advances and Applications of DSmT for Information Fusion, vol. 1, American Research Press, 2004.

[11] STANAG 1241 (2005). NATO Standard Identity Description Structure for Tactical Use, North Atlantic Treaty Organization, April 2005.

[12] P. Djiknavorian, and D. Grenier, Reducing DSmT hybrid rule complexity through optimisation of the calculation algorithm, in Advances and Applications of DSmT for Information Fusion, Collected Works edited by F. Smarandache, J. Dezert, Volume 2, American Research Press, 2006.

[13] F. Smarandache, J. Dezert, editors. Advances and Applications of DSmT for Information Fusion, vol. 2, ARP, 2006.

[14] P. Djiknavorian, Fusion d'informations dans un cadre de raisonnement de Dezert-Smarandache appliquée sur des rapports de capteurs ESM sous le STANAG 1241, Mémoire de maîtrise, Université Laval, 2008.

[15] P. Djiknavorian, P. Valin, and D. Grenier, Dezert-Smarandache theory applied to highly conflicting reports for identification and recognition - Illustrative example of ESM associations in dense environments, DRDC Valcartier TR 2008- 537, 34 pages.

[16] P. Djiknavorian, P. Valin, and D. Grenier, Fusion of ESM allegiance reports using DSmT, in Advances and Applications of DSmT for Information Fusion, Collected Works edited by F. Smarandache, J. Dezert, Volume 3, American Research Press, 2009.

[17] F. Smarandache, J. Dezert, editors. Advances and Applications of DSmT for Information Fusion, vol. 3, American Research Press, 2009.

[18] T. Carroll, J. Cooper and P. Tetali, Counting Antichains and Linear Extensions in Generalizations of the Boolean Lattice, August 30, 2009. http://www.math.sc.edu/ cooper/calegbl.pdf

[19] Lowrance, J. D., Garvey, T. D., and Strat, T. M., A framework for evidential reasoning systems, Proceedings of the 5th National Conference of the American Association for Artificial Intelligence, Philadelphia, 896-903, Aug. 1986. 